\title{
FONTES E DOSES DE FÓSFORO NO DESENVOLVIMENTO E PRODUÇÃO DO CAFEEIRO, EM UM SOLO ORIGINALMENTE SOB VEGETAÇÃO DE CERRADO DE PATROCÍNIO - MG
}

\author{
Sources and doses of phosphorus on coffee development and production on soil originally under \\ savannah vegetation of Patrocínio - MG
}

\author{
Benjamim de Melo ${ }^{1}$, Karina Velini Marcuzzo ${ }^{2}$, Reges Eduardo Franco Teodoro ${ }^{1}$, Hudson de Paula Carvalho ${ }^{3}$ \\ RESUMO
}

Conduziu-se este trabalho com o objetivo de avaliar o efeito de diferentes fontes e doses de fósforo no desenvolvimento e na produção do cafeeiro, cultivar Acaiá Cerrado, linhagem MG-1474, em um Latossolo Vermelho distroférrico, da Fazenda Experimental de Patrocínio, da Empresa de Pesquisa Agropecuária de Minas Gerais (EPAMIG). O experimento foi instalado no espaçamento de 3,50 × 0,70 m, segundo o delineamento experimental de blocos casualizados, em esquema fatorial 4 x 5 , com quatro repetições. Utilizaram-se como fontes de fósforo, o fosfato de Araxá, o termofosfato magnesiano, o fosfato de Arad e o superfosfato triplo, aplicados em cinco doses, correspondentes a: 0 (zero), 125, 250, 500 e 1.000 g de $\mathrm{P}_{2} \mathrm{O}_{5}$ total por metro de sulco. Cada parcela experimental foi constituída por uma linha com oito plantas, sendo adotadas como plantas úteis as quatro centrais. Aos trinta e aos quarenta e um meses após o plantio foram avaliadas as seguintes características: altura de planta (m), diâmetros de caule (mm) e de copa (m), e produtividade (sc/ha). As fontes de fósforo testadas comportaram-se de forma semelhante quanto às características de desenvolvimento do cafeeiro, aos 30 e aos 41 meses após o plantio; aos 30 meses, as maiores produtividades foram obtidas quando se utilizou o superfosfato triplo e o termofosfato magnesiano; aos 41 meses, as maiores produtividades foram observadas quando se utilizou os fosfatos de Araxá, de Arad e o termofosfato magnesiano; as doses de $\mathrm{P}_{2} \mathrm{O}_{5}$ influenciaram o desenvolvimento vegetativo do cafeeiro, sendo os melhores resultados observados na faixa compreendida pelas doses 618,8 a 674,4 g de $\mathrm{P}_{2} \mathrm{O}_{5}$ por metro de sulco; as melhores produtividades, foram obtidas nas doses compreendidas entre 539,7 a 855,0 g de $\mathrm{P}_{2} \mathrm{O}_{5}$ por metro de sulco, de acordo com o fertilizante utilizado, à exceção do superfosfato triplo, aos 41 meses após o plantio, em que a dose máxima de $\mathrm{P}_{2} \mathrm{O}_{5}$ testada foi insuficiente para se obter um máximo de produtividade.

TERMOS PARA INDEXAÇÃO: Coffea arabica, adubação fosfatada, fosfato natural, fosfato reativo.

\begin{abstract}
The objective of this work was to evaluate the effect of different sources and doses of phosphorus on coffee plant development and production, Acaiá Cerrado cultivate, MG 1474-lineage, on Distroferric Red Latosol, of Patrocínio Experimental Farm, belonging to EPAMIG. The experiment was installed on spacement of $3.50 \times 0.70 \mathrm{~m}$, in a randomized-block design, in a factorial 4 x 5, with four repetitions. The phosphorus sources were: “Araxá” phosphate, magnesium thermophosphate, Arad phosphate and triple superphosphate, all of them applied in five doses, corresponding to: 0 (zero), 125, 250, 500 and 1,000 g of $\mathrm{P}_{2} \mathrm{O}_{5}$ per meter of furrow. Each experimental plot was constituted by a line with eight plants, being used the four central ones as useful plants. Thirty and forty-one months after planting were evaluated the following characteristics: plant height, stem and canopy diameters and yield. The phosphorus sources had a similar behavior in relation to coffee development, 30 and 41 months after planting; 30 months after planting, the biggest yields were obtained when the triple superphosphate and the magnesium termophosphate were used; at 41 months, the biggest yields were obtained when the "Araxá” phosphate, the Arad phosphate and the magnesium termophosphate were used; the $\mathrm{P}_{2} \mathrm{O}_{5}$ doses influenced the coffee vegetative development, the best results were observed from 618,8 to $674,4 \mathrm{~g}$ of $\mathrm{P}_{2} \mathrm{O}_{5}$ per meter of furrow; the best yields were obtained with the doses from 539,7 to $855,0 \mathrm{~g}$ of $\mathrm{P}_{2} \mathrm{O}_{5}$ per meter of furrow, according to the used fertilizer, with exception of triple superphosphate, 41 months after planting, where the $\mathrm{P}_{2} \mathrm{O}_{5}$ maximum dose wasn't sufficient to obtain a maximum of yield.
\end{abstract}

INDEX-TERMS: Coffea arabica, Phosphorus manuring, natural phosphate, reative phosphate.

(Recebido para publicação em 14 de Janeiro de 2004 e aprovado em 17 de fevereiro de 2005)

\section{INTRODUÇÃO}

O café é um dos principais produtos de exportação do Brasil, cujo cultivo ocupa extensas áreas agrícolas do País, com destaque para a região do cerrado mi- neiro, pelas suas condições de solo, uma topografia ligeiramente plana e um clima, com um período seco na colheita, permitindo a obtenção de um café de melhor qualidade. A ocupação dessa área tem sido cada vez mais intensa ao longo dos anos.

\footnotetext{
1. Professor do Instituto de Ciências Agrárias - Universidade Federal de Uberlândia - Caixa Postal 593 - $38.400-902$ - Uberlândia, MG benjamim@umuarama.ufu.br

2. Mestre e bolsista do Consórcio Brasileiro de Pesquisa e Desenvolvimento/Café

3. Mestrando do Departamento de Engenharia - Universidade Federal de Lavras/UFLA - Caixa postal 3037 - 37.200-000 - Lavras, MG.
} 
MELO, B. de et al.

Os solos originalmente sob vegetação de Cerrado, entretanto, são muito intemperizados. Com o aumento do grau de intemperismo, há uma mudança gradual nas características desses solos, basicamente no sentido de torná-los menos eletronegativos. A capacidade de troca catiônica reduz, a adsorção aniônica aumenta, diminuindo a saturação por bases e aumentando gradualmente a retenção de ânions, como o fosfato (NOVAIS \& SMYTH, 1999). Estes solos são, dessa forma, extremamente pobres em fósforo (P) disponível. São necessárias aplicações de elevadas doses de fertilizantes fosfatados por ocasião do plantio, mas são extraídas pelas plantas quantidades relativamente pequenas de $\mathrm{P}$, indicando que grande parte dos fosfatos adicionados estaria indisponível para o cafeeiro em crescimento.

Nessas condições, a adubação fosfatada assume papel importante no sistema de produção cafeeira implantado em "solo de Cerrado". Barros et al., (2000) comprovaram a importância da aplicação do P no plantio do cafeeiro, sendo que nos tratamentos onde não se aplicou este nutriente na cova de plantio, observou-se apenas uma recuperação parcial da produtividade das plantas.

Garcia \& Ferreira (1997), estudando o fertilizante atifós (30\% de $\mathrm{P}_{2} \mathrm{O}_{5}$ total e $10,5 \%$ de $\mathrm{P}_{2} \mathrm{O}_{5}$ solúvel em ácido cítrico), em comparação com o superfosfato triplo (com $41 \%$ de $\mathrm{P}_{2} \mathrm{O}_{5}$, fósforo determinado como $\mathrm{P}_{2} \mathrm{O}_{5}$ solúvel em CNA + água e mínimo de $37 \%$ solúvel em água) e o fosfato de Araxá (24\% de $\mathrm{P}_{2} \mathrm{O}_{5}$ determinado como $\mathrm{P}_{2} \mathrm{O}_{5}$ total e mínimo de $4 \%$ solúvel em ácido cítrico a $2 \%$ na relação $1: 100$ ), nas doses de 30 e $60 \mathrm{~g}$ de $\mathrm{P}_{2} \mathrm{O}_{5} /$ cova, em solo com $1,3 \mathrm{mg}$ de $\mathrm{P} \mathrm{dm}^{-3}$, no espaçamento de 3,5 x 1,0 m, verificaram a importância do P na formação do cafeeiro, quando a produção obtida aumentou em até $286 \%$, aos 30 meses após o plantio. Estes autores relataram, ainda, que quando se aplicou as três fontes, na presença de calcário, tiveram comportamento semelhante para a dose maior de $\mathrm{P}_{2} \mathrm{O}_{5}$ (60 g/cova), sendo que na menor dose (30 g/cova) o fosfato de Araxá foi inferior, seguido do superfosfato triplo.

Barros et al., (2001) estudaram a influência de duas fontes de $\mathrm{P}$ (superfosfatos simples e triplo) nas doses de $0,100,200,400$ e $800 \mathrm{~kg}$ de $\mathrm{P}_{2} \mathrm{O}_{5} /$ ha, na formação e manutenção (após o $4^{\circ}$ ano do plantio, retomou-se a aplicação anual de $\mathrm{P}_{2} \mathrm{O}_{5}$ ) do cafeeiro em plantio super adensado (1,5 x 0,7 m), no município de Martins Soares - MG. O solo (Latossolo Vermelho Amarelo húmico) apresentava um teor médio de $\mathrm{P}$ de $1,9 \mathrm{mg} \mathrm{dm}^{-3}$; verificaram que as duas fontes apresentaram comportamento semelhante, no tipo de solo estudado e que a dose de $800 \mathrm{~kg}$ de $\mathrm{P}_{2} \mathrm{O}_{5} / \mathrm{ha}\left(120 \mathrm{~g}\right.$ de $\left.\mathrm{P}_{2} \mathrm{O}_{5} / \mathrm{m}\right)$, juntamente à adubação de manutenção após o $4^{0}$ ano, foi a que promoveu maior aumento de produção no plantio super adensado.

Santinato et al. (1998) avaliaram os efeitos de fontes e de doses de P na produção do cafeeiro, aos 36 meses de idade, em Latossolo Vermelho Amarelo de Carmo do Paranaíba - MG, com 1,0 mg de $\mathrm{P} \mathrm{dm}^{-3}$, e verificaram a importância deste nutriente nessa fase; todas as fontes estudadas e em todas as doses foram superiores à testemunha. Estes autores concluíram, ainda, que as melhores fontes dos fertilizantes em suas melhores doses foram o superfosfato simples e o fosmag, na dose de $400 \mathrm{~g}$, o superfosfato triplo, o Arad e o atifós, na dose de $300 \mathrm{~g}$ e o termofosfato magnesiano na dose de 200 g de $\mathrm{P}_{2} \mathrm{O}_{5}$ solúvel por metro de sulco .

Poucos têm sido, no entanto, os trabalhos realizados com o P na cafeicultura, visando avaliar quais as melhores fontes e doses dos fertilizantes fosfatados, assim como os efeitos que eles exercem sobre o desenvolvimento e produção do cafeeiro.

Objetivou-se com este trabalho foi avaliar o efeito de diferentes fontes e doses de P no desenvolvimento e produção do cafeeiro, cultivado em Latossolo Vermelho distroférrico, do município de Patrocínio - MG.

\section{MATERIAL E MÉTODOS}

O experimento foi conduzido em um solo originalmente sob vegetação de cerrado, da Fazenda Experimental de Patrocínio, da Empresa de Pesquisa Agropecuária de Minas Gerais - EPAMIG, localizada em Patrocínio, Minas Gerais, no período de janeiro de 2000 a julho de 2003. O solo da área experimental foi classificado como Latossolo Vermelho distroférrico (LVd), de acordo com a Empresa Brasileira de Pesquisa Agropecuária - EMBRAPA (1999). As análises físicas e químicas do solo da área experimental estão apresentadas nas Tabelas 1 e 2.

Foram utilizadas mudas da cultivar Acaiá Cerrado, linhagem MG-1474, formadas em sacos plásticos, que apresentavam 4 a 5 pares de folhas quando, após aclimatadas, foram plantadas em 10/01/2000. Nos sulcos previamente preparados e distanciados de $3,5 \mathrm{~m}$ abriram-se covetas para o plantio das mudas distanciadas de $0,70 \mathrm{~m}$.

$\mathrm{O}$ experimento foi instalado segundo o delineamento experimental de blocos casualizados, em esquema fatorial $4 \times 5$, com quatro repetições. Cada parcela experimental era constituída por uma linha com oito plantas, sendo adotadas como plantas úteis as quatro centrais. 
Fontes e doses de fósforo no desenvolvimento e produção do cafeeiro...

TABELA 1 - Análises físicas ${ }^{1}$ de amostras do solo nas profundidades de 0 a 20 e 20 a $40 \mathrm{~cm}$.

\begin{tabular}{ccccc}
\hline Profundidade & Areia grossa & Areia fina & Silte & Argila \\
\hline $0-20$ & 60 & 40 & 210 & 690 \\
$20-40$ & 40 & 30 & 190 & 740 \\
\hline
\end{tabular}

${ }^{1}$ Realizadas no Laboratório de Análises de Solos do Instituto de Ciências Agrárias da Universidade Federal de Uberlândia. (UFU), Uberlândia, MG, 2003.

TABELA 2 - Análises químicas ${ }^{1}$ de amostras do solo nas profundidades de 0 a 20 e 20 a $40 \mathrm{~cm}$.

\begin{tabular}{|c|c|c|c|c|c|c|c|c|}
\hline Profundidade & $\begin{array}{c}\text { pH em } \\
\text { água }\end{array}$ & $\mathbf{P}$ & $\mathbf{K}$ & Ca & Mg & $\mathbf{T}$ & $\mathbf{V}$ & $\mathbf{M}$ \\
\hline --------cm------- & $(1: 2,5)$ & \multicolumn{2}{|c|}{--------mg dm ${ }^{-3}$------- } & \multicolumn{2}{|c|}{----- $\mathrm{cmol}_{(\mathrm{c})} \mathrm{dm}^{-3}-----$} & - & $---\%-$ & \\
\hline $0-20$ & 5,20 & 0,3 & 74,2 & 0,5 & 0,2 & 5,88 & 14 & 11 \\
\hline $20-40$ & 5,20 & 0,1 & 31,5 & 0,2 & 0,0 & 4,84 & 7 & 24 \\
\hline
\end{tabular}

${ }^{1}$ Realizadas no Laboratório de Análises de Solos do Instituto de Ciências Agrárias da Universidade Federal de Uberlândia. (UFU), Uberlândia, MG, 2003.

Como tratamentos foram utilizadas quatro fontes de fósforo, o fosfato de Araxá, com $24 \%$ de $\mathrm{P}_{2} \mathrm{O}_{5}$ determinado como $\mathrm{P}_{2} \mathrm{O}_{5}$ total e mínimo de $4 \%$ solúvel em ácido cítrico a $2 \%$ na relação $1: 100$ e 23 a $27 \%$ de Ca; termofosfato magnesiano, com $17 \%$ de $\mathrm{P}_{2} \mathrm{O}_{5}$ determinado como $\mathrm{P}_{2} \mathrm{O}_{5}$ total e mínimo de $14 \%$ solúvel em ácido cítrico a $2 \%$ na relação 1:100 e, $7 \%$ de $\mathrm{Mg}$ e 18 a 20\% de Ca; fosfato de Arad, com $33 \%$ de $\mathrm{P}_{2} \mathrm{O}_{5}$ determinado como $\mathrm{P}_{2} \mathrm{O}_{5}$ total e mínimo de $10,5 \%$ solúvel em ácido cítrico a $2 \%$ na relação $1: 100$ e $37 \%$ de Ca e o superfosfato triplo, com $41 \%$ de $\mathrm{P}_{2} \mathrm{O}_{5}$, fósforo determinado como $\mathrm{P}_{2} \mathrm{O}_{5}$ solúvel em CNA + água e mínimo de $37 \%$ solúvel em água e 12 a $14 \%$ de $\mathrm{Ca}$, todas elas aplicadas em cinco doses de $\mathrm{P}_{2} \mathrm{O}_{5}(0,125,250,500$ e $1.000 \mathrm{~g}$ de $\mathrm{P}_{2} \mathrm{O}_{5}$ total/m de sulco). As doses dos fertilizantes fosfatados foram calculadas com base nos teores de $\mathrm{P}_{2} \mathrm{O}_{5}$ total de cada fonte.

Aplicou-se calcário em área total, para elevar a saturação por bases para $60 \%$, correspondendo à aplicação de $2.700 \mathrm{~kg}$ de calcário dolomítico/ha, com $100 \%$ de Poder Relativo de Neutralização Total (PRNT). Foram realizadas uma aração e duas gradagens e, em seguida, os sulcos foram abertos a $40 \mathrm{~cm}$ de profundidade e $60 \mathrm{~cm}$ de largura, em forma de V, e então aplicadas as diferentes fontes e doses de fósforo, conforme os tratamentos considerados. Utilizou-se um subsolador de duas hastes para incorporar as fontes no solo.
As adubações de plantio e pós-plantio, para o nitrogênio e o potássio, e foliares (micronutrientes) foram realizadas segundo recomendações da CFSEMG (1999). No ano de 2000, foram realizadas três adubações de cobertura, em pós-plantio, aplicando-se $15 \mathrm{~g}$ de $\mathrm{K}_{2} \mathrm{O}$ /planta e 4 g de $\mathrm{N}$ /planta, utilizando-se o cloreto de potássio e o sulfato de amônio como fontes. Realizaram-se, ainda, quatro adubações foliares com micronutrientes, aplicando-se, 0,2\% de Cobre Sandoz BR, 0,3\% de ácido bórico, 0,8\% de sulfato de zinco, 0,6\% de sulfato manganoso, $0,25 \%$ de cloreto de potássio e $0,8 \%$ de melaço em pó. Nas pulverizações foliares acrescentou-se, ainda, defensivos para o controle de ferrugem e bicho-mineiro, quando necessário. No primeiro ano pós-plantio (2001), realizaram-se três aplicações de nitrogênio e potássio. Na primeira, utilizaram-se $4 \mathrm{~g}$ de $\mathrm{N}$ /planta, na forma de sulfato de amônio e $15 \mathrm{~g}$ de $\mathrm{K}_{2} \mathrm{O}$ /planta, na forma de cloreto de potássio. Na segunda e na terceira aplicação, utilizaram-se o formulado 20-0020, na quantidade de $125 \mathrm{~g}$ do produto/m de sulco. Foram realizadas, ainda, quatro pulverizações com micronutrientes e potássio, nas mesmas concentrações citadas anteriormente, aliando-se a aplicação de defensivos, quando necessário. No segundo ano pós-plantio (2002) realizaram-se quatro adubações de cobertura, aplicandose $125 \mathrm{~g}$ do formulado $20-00-20 / \mathrm{m}$ de sulco e 5 adubações foliares com micronutrientes, sendo que nas três 
primeiras as concentrações foram iguais às feitas nos anos anteriores e, nas duas últimas, com as seguintes concentrações: $0,5 \%$ de uréia, $0,4 \%$ de ácido bórico, $0,3 \%$ de sulfato de zinco, $0,3 \%$ de cloreto de potássio, 0,3\% de sulfato manganoso, $0,5 \%$ de sulfato cuproso e 0,5\% de cal. No terceiro ano pós-plantio (2003), até o período em que foi realizada a avaliação do experimento, realizou-se uma adubação em cobertura, na dose de $125 \mathrm{~g}$ do formulado $20-00-20 / \mathrm{m}$ de sulco e duas pulverizações foliares nas concentrações citadas anteriormente.

Aos trinta e aos quarenta e um meses após o plantio, foram avaliadas as seguintes características: altura de planta $(\mathrm{m})$, diâmetro de caule $(\mathrm{mm})$, diâmetro de copa (m) e produtividade (sc/ha).

Os dados obtidos foram submetidos à análises estatísticas apropriadas ao modelo experimental adotado, procedendo-se a análise de variância, com aplicação do teste de F, nos níveis de significância de 1 e 5\% de probabilidade. Resultados significativos para o fator quantitativo (doses de fósforo) foram submetidos à análise de regressão e, para o fator qualitativo (fontes de fósforo), as médias foram comparadas com a aplicação do teste de Tukey, ao nível de 5\% de probabilidade, conforme Gomes (1966).

Foram calculados os pontos de máximo e de mínimo, para as regressões quadráticas, utilizando-se derivadas.

\section{RESULTADOS E DISCUSSÃO}

Pela análise de variância dos dados observaram-se diferenças significativas entre as fontes de fósforo e a interação fontes $\mathrm{x}$ doses, aos trinta meses e aos quarenta e um meses após o plantio, apenas para a produtividade do cafeeiro; as doses de fósforo diferiram significativamente em todas as características analisadas, nos dois períodos, à exceção da altura de plantas, aos 30 meses após o plantio. As análises das características avaliadas, em função das fontes de fósforo, podem ser observadas na Tabela 3. Verifica-se que, aos trinta meses após o plantio, as maiores produtividades foram obtidas com a utilização do termofosfato magnesiano (25,96 sc/ha) e do superfosfato triplo (26,07 sc/ha), fertilizantes, cujas quantidades aplicadas, apesar de semelhantes entre si e também às outras fontes, apresentaram um maior teor de $\mathrm{P}_{2} \mathrm{O}_{5}$ solúvel prontamente disponível às plantas. Os resultados obtidos concordam, em parte, com os de Santinato et al. (1998), que verificaram que as maiores produtividades do cafeeiro, aos 36 meses após o plantio, foram obtidas quando se utilizaram o termofosfato magnesiano, superfosfato triplo, fosfato de Arad, super- fosfato simples, fosmag e atifós, sendo estas fontes superiores ao fosfato de Araxá. Aos quarenta e um meses após o plantio, entretanto, as maiores produtividades foram obtidas quando se utilizou o fosfato de Araxá (13,15 sc/ha), o fosfato de Arad (11,51 sc/ha) e o termofosfato magnesiano (11,19 sc/ha), que não diferiram entre si; a menor produtividade foi observada quando se utilizou o superfosfato triplo (8,88 sc/ha). Estes resultados podem ser explicados pela solubilidade que apresentam os fertilizantes utilizados. O superfosfato triplo, por ser uma fonte de fósforo solúvel, disponibiliza imediatamente seus nutrientes para a solução do solo, proporcionando maiores produtividades na primeira avaliação. O termofosfato magnesiano, por apresentar uma porcentagem de $\mathrm{P}$ solúvel de cerca de $17 \%$, e outra, disponibilizada de maneira gradual, proporcionou altas produtividades nos dois períodos de avaliação e os fosfatos de Araxá e de Arad, por se tratarem de fontes de fósforo menos solúveis, disponibilizaram este nutriente ao cafeeiro gradualmente, como pode ser observado na segunda avaliação, em que as maiores produtividades foram observadas com a utilização destes fertilizantes.

A análise de regressão para os diâmetros de caule e de copa e a produtividade, em função das doses de fósforo, aos trinta e aos quarenta e um meses após o plantio, e da altura de plantas aos quarenta e um meses, encontram-se nas Figuras 1 a 5, respectivamente. Podese verificar que os maiores valores de diâmetros de caule, aos 30 e aos 41 meses após o plantio, foram obtidos com as doses de 618,8 e de 674,4 g de $\mathrm{P}_{2} \mathrm{O}_{5} / \mathrm{m}$ de sulco, respectivamente (Figura 1). Os maiores diâmetros de copa, aos 30 e aos 41 meses após o plantio, foram obtidos com as doses de 620,8 e de 643,2 g de $\mathrm{P}_{2} \mathrm{O}_{5} / \mathrm{m}$ de sulco (Figura 2). Aos 30 meses após o plantio a maior produtividade, quando se utilizou o superfosfato triplo, o termofosfato magnesiano, o fosfato de Araxá e o de Arad foi obtida, respectivamente nas doses de 855,0; 620,$8 ; 572,0$ e 581,0 g de $\mathrm{P}_{2} \mathrm{O}_{5} / \mathrm{m}$ de sulco (Figura 3 ). Aos 41 meses, a maior produtividade quando se utilizou o termofosfato magnesiano foi obtida com a dose de 579,4 g; para o fosfato de Araxá foi obtida com a dose de 711,7 g; para o fosfato de Arad, com a dose de 539,7 $\mathrm{g}$ de $\mathrm{P}_{2} \mathrm{O}_{5} / \mathrm{m}$ de sulco e, para o superfosfato triplo, a produtividade foi crescente com o aumento das doses de $\mathrm{P}_{2} \mathrm{O}_{5}$ (Figura 4).

Os dados mostram que apesar das doses de $\mathrm{P}_{2} \mathrm{O}_{5}$ solúvel nos fosfatos de baixa solubilidade terem sido menores que nos adubos mais solúveis, com o passar do tempo os fosfatos naturais passaram a solubilizar $\mathrm{P}$ e os fosfatos solúveis foram fixando $\mathrm{P}$ e ficando menos dis- 
poníveis. Os resultados obtidos neste trabalho concordam com os de Garcia \& Ferreira (1997), em que verifica-se a importância da adubação fosfatada de plantio na produtividade do cafeeiro, tendo as doses de todos os fertili- zantes utilizados resultado em produtividades superiores à da testemunha (dose zero). Observa-se na Figura 5, que a maior altura de plantas, aos 41 meses após o plantio, foi obtida com a dose de 628,1 g de $\mathrm{P}_{2} \mathrm{O}_{5}$.

TABELA 3 - Resultados médios ${ }^{1}$ para as características do cafeeiro avaliadas no experimento, aos trinta e aos quarenta e um meses após o plantio, em função das diferentes fontes de fósforo. (UFU), Uberlândia, MG, 2003.

\begin{tabular}{|c|c|c|c|c|c|c|c|c|}
\hline \multirow[t]{2}{*}{ Fontes de $\mathrm{P}_{2} \mathrm{O}_{5}$} & \multicolumn{2}{|c|}{$\begin{array}{c}\text { Altura de } \\
\text { Planta }\end{array}$} & \multicolumn{2}{|c|}{$\begin{array}{c}\text { Diâmetro de } \\
\text { Caule }\end{array}$} & \multicolumn{2}{|c|}{$\begin{array}{c}\text { Diâmetro de } \\
\text { copa }\end{array}$} & \multicolumn{2}{|c|}{ Produtividade } \\
\hline & \multicolumn{2}{|c|}{ (m) } & \multicolumn{2}{|c|}{$(\mathbf{m m})$} & \multicolumn{2}{|c|}{ (m) } & \multicolumn{2}{|l|}{ (sc/ha) } \\
\hline Fosfato de Araxá & 1,45 & $\mathrm{a}$ & 38,94 & $\mathrm{a}$ & 1,34 & $\mathrm{a}$ & 20,07 & $\mathrm{~b}$ \\
\hline Termofosfato magnesiano & 1,43 & $\mathrm{a}$ & 40,10 & $\mathrm{a}$ & 1,36 & $\mathrm{a}$ & 25,96 & $\mathrm{a}$ \\
\hline Fosfato de Arad & 1,45 & $\mathrm{a}$ & 39,75 & $\mathrm{a}$ & 1,39 & $\mathrm{a}$ & 20,28 & $\mathrm{~b}$ \\
\hline Superfosfato Triplo & 1,42 & $\mathrm{a}$ & 40,50 & $\mathrm{a}$ & 1,34 & $\mathrm{a}$ & 26,07 & $\mathrm{a}$ \\
\hline & -- & & - & & ------- & 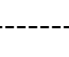 & ------- & \\
\hline Fosfato de Araxá & 1,8 & $\mathrm{a}$ & 50,00 & $\mathrm{a}$ & 1,57 & $\mathrm{a}$ & 13,15 & $\mathrm{a}$ \\
\hline Termofosfato magnesiano & 1,9 & $\mathrm{a}$ & 51,62 & $\mathrm{a}$ & 1,65 & $\mathrm{a}$ & 11,19 & $\mathrm{ab}$ \\
\hline Fosfato de Arad & 1,9 & $\mathrm{a}$ & 52,61 & $\mathrm{a}$ & 1,63 & $\mathrm{a}$ & 11,51 & $\mathrm{ab}$ \\
\hline Superfosfato Triplo & 1,90 & $\mathrm{a}$ & 52,00 & $\mathrm{a}$ & 1,58 & $\mathrm{a}$ & 8,88 & $\mathrm{~b}$ \\
\hline
\end{tabular}

${ }^{1}$ Médias seguidas de mesma letra na vertical, em cada época de avaliação, não diferem entre si, ao nível de 5\% de probabilidade, pelo teste de Tukey.

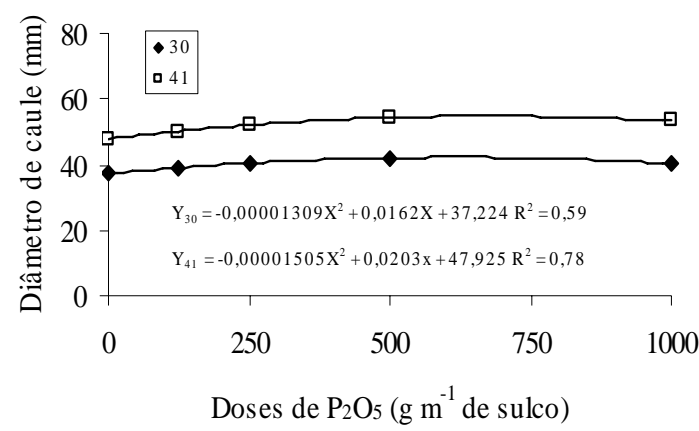

FIGURA 1 - Regressão dos valores médios de diâmetro de caule $(\mathrm{mm})$ em função das doses de $\mathrm{P}_{2} \mathrm{O}_{5}$ total ( $\mathrm{g} \mathrm{m}^{-1}$ de sulco) aos 30 e aos 41 meses após o plantio. (UFU), Uberlândia, MG, 2003.

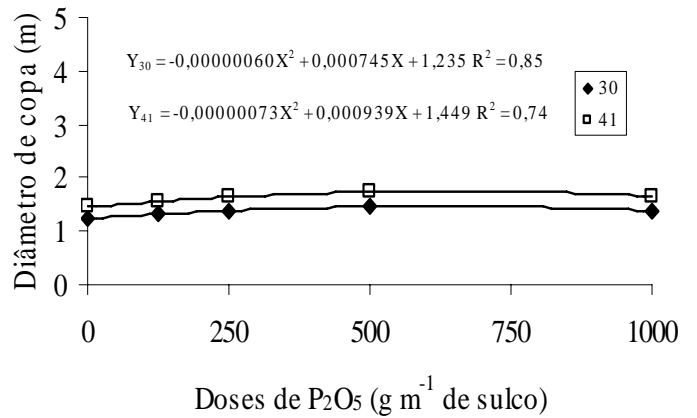

FIGURA 2 - Regressão dos valores médios de diâmetro de copa (m) em função das doses de $\mathrm{P}_{2} \mathrm{O}_{5}$ total $\left(\mathrm{g} \mathrm{m}^{-1}\right.$ de sulco) aos 30 e aos 41 meses após o plantio. (UFU), Uberlândia, MG, 2003. 


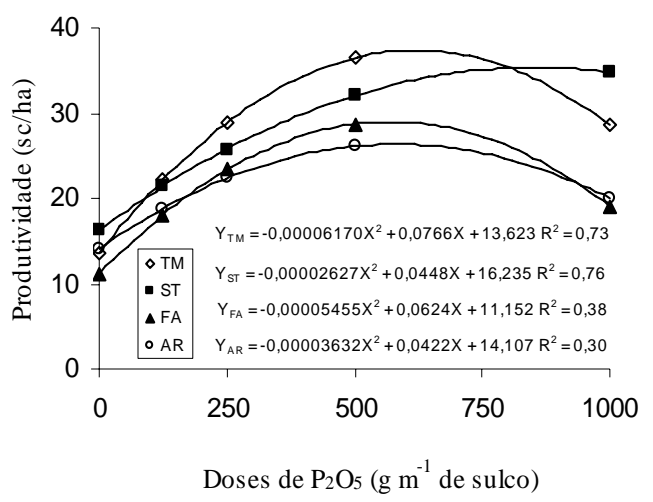

FIGURA 3 - Regressão dos valores médios de produtividade em função das doses de $\mathrm{P}_{2} \mathrm{O}_{5}$ total $\left(\mathrm{g} \mathrm{m}^{-1}\right.$ de sulco) aos 30 meses após o plantio. (UFU), Uberlândia, MG, 2003.

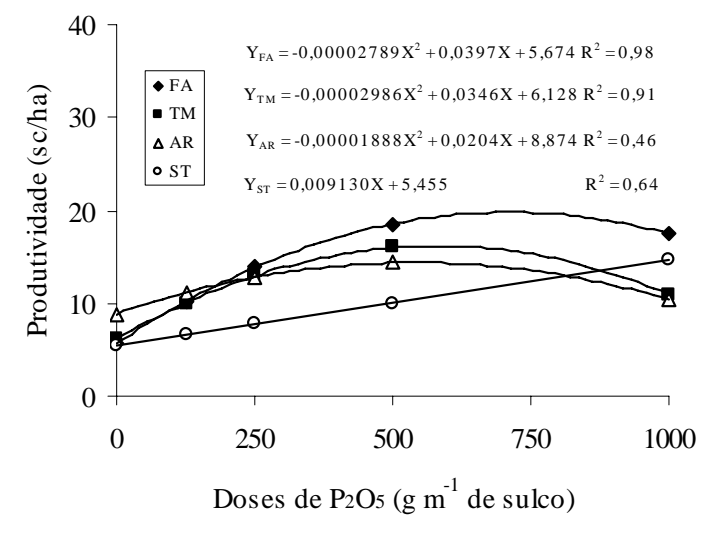

FIGURA 4 - Regressão dos valores médios de produtividade em função das doses de $\mathrm{P}_{2} \mathrm{O}_{5}$ total ( $\mathrm{g} \mathrm{m}^{-1}$ de sulco) aos 41 meses após o plantio. (UFU), Uberlândia, MG, 2003.

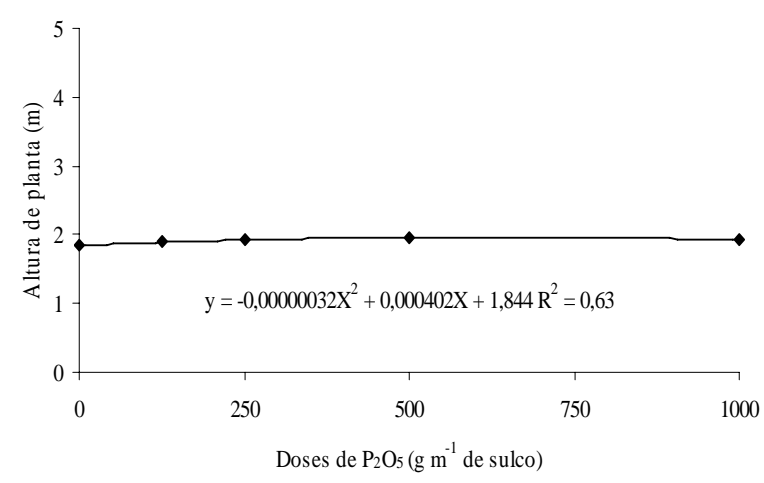

FIGURA 5 - Regressão dos valores médios de altura de planta em função das doses de $\mathrm{P}_{2} \mathrm{O}_{5}$ total (g m ${ }^{-1}$ de sulco), aos 41 meses após o plantio. (UFU), Uberlândia, MG, 2003.

\section{CONCLUSÕES}

Nas condições em que o trabalho foi conduzido, os dados obtidos para a cultivar Acaiá Cerrado, linhagem MG 1474, permitem concluir que:

As fontes de fósforo testadas comportaram-se de forma semelhante quanto às características de desenvolvimento do cafeeiro, aos 30 e aos 41 meses após o plantio.

Aos 30 meses após o plantio as maiores produtividades foram obtidas quando se utilizou o superfosfato triplo e o termofosfato magnesiano; aos 41 meses, as maiores produtividades foram observadas quando se utilizou os fosfatos de Araxá e de Arad e o termofosfato magnesiano.

As doses de $\mathrm{P}_{2} \mathrm{O}_{5}$ influenciaram o desenvolvimento vegetativo do cafeeiro, sendo os melhores resultados observados na faixa de 618,8 a 674,4 g de $\mathrm{P}_{2} \mathrm{O}_{5} / \mathrm{m}$ de sulco.

As melhores produtividades foram obtidas com as doses de 539,7 a 855,0 g de $\mathrm{P}_{2} \mathrm{O}_{5} / \mathrm{m}$ de sulco, de acordo com o fertilizante utilizado, à exceção do superfosfato triplo, aos 41 meses após o plantio, em que a dose máxima de $\mathrm{P}_{2} \mathrm{O}_{5}$ foi insuficiente para se obter uma produtividade máxima. 
Sugere-se aplicar metade da quantidade de $\mathrm{P}_{2} \mathrm{O}_{5}$ com uma fonte solúvel e metade com uma fonte de baixa solubilidade.

\section{REFERÊNCIAS BIBLIOGRÁFICAS}

BARROS, U. V. et al. Recuperação de cafeeiros com aplicação corretiva de adubo fosfatado em plantas sem fósforo na cova de plantio. In: CONGRESSO BRASILEIRO DE PESQUISAS CAFEEIRAS, 26., 2000, Marília. Resumos... Rio de Janeiro: IBG/GERCA, 2000. p. 64-66.

BARROS, U. V. et al. Doses e fontes de fósforo em cafeeiros super adensados em solo LVAh na Zona da Mata de Minas Gerais. In: CONGRESSO BRASILEIRO DE PESQUISAS CAFEEIRAS, 27., 2001, Uberaba. Resumos... Rio de Janeiro: IBG/GERCA, 2001. p. 27-28.

COMISSÃO DE FERTILIDADE DO SOLO DO ESTADO DE MINAS GERAIS. Recomendações para o uso de corretivos e fertilizantes em Minas Gerais: 5ª aproximação. Viçosa, 1999.
EMPRESA BRASILEIRA DE PESQUISA AGROPECUÁRIA. Sistema brasileiro de classificação de solos. Brasília, 1999. 412 p.

GARCIA, A. W. R.; FERREIRA, R. A. Estudos comparativos de doses de Atifós e outras fontes de $\mathrm{P}_{2} \mathrm{O}_{5}$ na formação do cafeeiro - Varginha - MG. In: CONGRESSO BRASILEIRO DE PESQUISAS CAFEEIRAS, 23., 1997, Manhuaçu. Resumos... Rio de Janeiro: IBG/GERCA, 1997. p. 4-5.

GOMES, F. P. Curso de estatística experimental. Piracicaba, ESALQ, 1966. 404 p.

NOVAIS, R. F.; SMYTH, T. J. Fósforo em solo e planta em condições tropicais. Viçosa: UFV, 1999. $399 \mathrm{p}$.

SANTINATO, R.; FERNANDES, A. L. T.; PEREIRA, E. M. Fontes e doses crescentes de $\mathrm{P}_{2} \mathrm{O}_{5}$ (fósforo) na formação do cafeeiro em solo de cerrado. In: CONGRESSO BRASILEIRO DE PESQUISAS CAFEEIRAS, 24., 1998, Poços de Caldas. Resumos... Rio de Janeiro: IBG/GERCA, 1998. p. 93-94. 